\title{
Girls, Boys, and Bots: Gender Differences in Young Children's Performance on Robotics and Programming Tasks
}

\author{
Amanda Sullivan and Marina Umaschi Bers \\ Tufts University, Medford, Massachusetts, USA
}

\author{
Amanda.sullivan@tufts.edu; Marina.Bers@tufts.edu
}

\begin{abstract}
Prior work demonstrates the importance of introducing young children to programming and engineering content before gender stereotypes are fully developed and ingrained in later years. However, very little research on gender and early childhood technology interventions exist. This pilot study looks at $N=45$ children in kindergarten through second grade who completed an eight-week robotics and programming curriculum using the KIWI robotics kit. KIWI is a developmentally appropriate robotics construction set specifically designed for use with children ages 4 to 7 years old. Qualitative pre-interviews were administered to determine whether participating children had any gender-biased attitudes toward robotics and other engineering tools prior to using KIWI in their classrooms. Post-tests were administered upon completion of the curriculum to determine if any gender differences in achievement were present. Results showed that young children were beginning to form opinions about which technologies and tools would be better suited for boys and girls. While there were no significant differences between boys and girls on the robotics and simple programming tasks, boys performed significantly better than girls on the advanced programming tasks such as, using repeat loops with sensor parameters. Implications for the design of new technological tools and curriculum that are appealing to boys and girls are discussed.
\end{abstract}

Keywords: early childhood, robotics, programming, gender

\section{Introduction}

In fields like computer science and numerous other Science, Technology, Engineering, Mathematics (STEM) disciplines, men continue to outnumber women (Hill, Corbett, \& St. Rose, 2010). This persistent gender disparity may be due to the negative effect of stereotype threat on women's

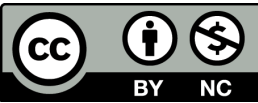

This article has been copyrighted by the Informing Science Institute on behalf of its authors. We license it to you under a Creative Commons AttributionNonCommercial 4.0 International License. When you copy and redistribute this paper in full or in part, you need to provide proper attribution to it to ensure that others can later locate this work (and to ensure that others do not accuse you of plagiarism). You may (and we encourage you to) adapt, remix, transform, and build upon the material for any non-commercial purposes. This license does not permit you to use this material for commercial purposes. confidence and interest in these traditionally masculine fields (Spencer, Steele, \& Quinn, 1999). One way to address this gender disparity is to attract the interest of girls during their formative early childhood before extreme gender stereotypes are ingrained (Metz, 2007; Steele, 1997). ). By age 5, children are already beginning to develop a range of stereotypes about gender and applying them to themselves and others (Martin \& Ruble, 2004). During the kindergarten through second grade years (ages 5-7) children are developing strict "all or 
nothing" views about gender making early childhood an important time for children to see that both boys and girls can be successful and competent in STEM areas. Robotics can be a playful way to engage young children (both boys and girls) in STEM content during this pivotal time in development. However, a lack of developmentally appropriate robotic tools for young children in engineering and programming makes this a challenge for parents and educators.

This paper presents results from the Ready for Robotics project, funded by the National Science Foundation, which focused on creating a developmentally appropriate robotics kit for young children aged four to seven years. While the majority of research on robotics and programming in education focuses on later schooling, teaching these subjects during foundational early childhood

years can be an engaging and rewarding experience for young learners and a potential way to dispel masculine stereotypes around robotics from forming (Sullivan \& Bers, 2013). Previous research has shown that children as young as four years old can build and program simple robotics projects (Bers, Ponte, Juelich, Viera, \& Schenker, 2002; Cejka, Rogers, \& Portsmore, 2006; Perlman, 1976; Wyeth, 2008). Additionally, robotic manipulatives allow children to work on skills that are important for healthy child development such as fine motor skills and hand-eye coordination while also engaging in collaboration and teamwork. They also provide a concrete and tangible way to understand abstract programming ideas such as repeat loops and conditional statements, because the child can directly view the impact of his or her programming commands on the robot's actions (Bers, 2008; Sullivan, Kazakoff, \& Bers, 2013). Moreover, introducing robotics and programming in early childhood may also serve as a way to increase girls' interest and abilities in robotics and computer science fields before ingrained stereotypes make this more difficult in later years (Metz, 2007; Steele, 1997).

The purpose of this study was to analyze results from the Ready for Robotics project in order to determine what (if any) gender stereotypes about technology and engineering young children already have beginning in Kindergarten and whether boys and girls were equally successful in their mastery of introductory robotics and programming concepts using a kit specifically designed for young children. This analysis will shed light on designing both tools and curriculum that are appealing and educational for both genders.

\section{Literature Review}

\section{The STEM Gender Gap}

The issue of girls' and women's underrepresentation in STEM (science, technology, engineering, and mathematics) fields has been a major area of concern to educators and researchers over the past 50 years (Hughes, Nzekwe, \& Molyneaux, 2013). The gender disparity between women and men in many STEM fields has noticeably decreased over the past decade; however, there are still several gaps that persist particularly when it comes to technology and engineering (Hill et al., 2010). For example, in Computer Science, female participation has been on a steady decline during the past decade (National Center for Women and Informational Technology, 2011). In 2009, only $11 \%$ of undergraduate Computer Science degrees from major research universities were granted to women and, between the years 2000-2009, there has been a 79\% decline in first year undergraduate women interested in pursuing Computer Science (National Center for Women and Informational Technology, 2011).

One reason men outnumber women in college Computer Science and related majors is that girls are less likely to take Advanced Placement (AP) exams in high school that could help prepare them to enter these majors. In high school, girls are less likely than boys to take numerous college preparatory science and math AP Exams including: Calculus, Computer Science, and Statistics (Hill et al., 2010). In the professional arena, women make up less than $30 \%$ of environmental sci- 
entists, less than $30 \%$ of computer scientists, less than $10 \%$ of electrical engineers, and less than $7 \%$ of mechanical engineers (Hill et al., 2010).

When it comes to robotics and computer programming, prior research has also demonstrated gender differences. Nourbakhsh, Hammer, Crowley, and Wilkinson (2004) looked at gender differences over a 7 week robotics course for high school students and found that girls were more likely to have struggled with programming than boys and that girls entered the course with less confidence than boys. Despite this, it was also found that by the end of the course girls' confidence increased more than the boys' did (Nourbakhsh et al., 2004). Similarly, Milto, Rogers, and Portsmore (2002) found that although men and women in an introductory engineering class displayed equivalent competency in robotics activities, men were more confident in their abilities than women.

\section{Stereotype Threat and STEM Identity}

STEM identification reflects the extent to which students view themselves as members of STEMrelated communities of practice (Aschbacher, Li, \& Roth, 2010). A STEM identity is informed by students' own perceptions of who they are and who they want to become with respect to STEM (Brickhouse \& Potter, 2001). However, the likelihood that girls and women will develop a strong STEM identity can be influenced by the negative effect of stereotype threat on their confidence and interest in these traditionally masculine areas. Stereotype threat refers to the anxiety that one's performance on a task or activity will be seen through the lens of a negative stereotype (Steele, 1997; Spencer et al., 1999). For example, Spencer et al. (1999) found that when women were shown gender differences on a math test (to induce stereotype threat) before being asked to complete it, they performed significantly worse than their male counterparts. When stereotype threat was not triggered (by telling participants that there were no gender differences associated with the test) women and men performed similarly on the test.

Stereotype threat is not only triggered by explicit statements. Environmental and situational factors (i.e., being in a certain place, interactions with a person, etc.) can also trigger a negative stereotype (Shapiro \& Williams, 2011). These implicit stereotypes may be learned through past experiences or behaviors and can persist even if an individual resists the stereotype explicitly. For example, we can have an unconscious stereotype that boys are better at math than girls even if consciously we do not necessarily believe this to be true. When we see a classmate who is female fail a math test or struggle with math homework, our unconscious stereotype may be triggered.

\section{Robotics and Programming in Early Childhood}

Research suggests that children who are exposed to STEM curriculum and programming at an early age demonstrate fewer gender-based stereotypes regarding STEM careers (Metz, 2007; Steele, 1997) and fewer obstacles entering these fields later in life (Madill et al., 2007; Markert, 1996). These types of early interventions could be one way to effectively avoid long-lasting negative stereotypes and allow young girls to begin fostering a STEM identity from early childhood.

Although educational robotics kits are more often seen in middle and high school environments, robotics offers an engaging way to teach young children about the types of electronics and sensors they encounter in daily life. Teaching foundational programming concepts, along with robotics, makes it possible to introduce young children to important ideas that inform the design of many of the everyday objects everyday objects with which they interact (Bers, 2008). Moreover, introducing robotics and computer programming in early childhood may give young girls a chance to positively engage with engineering before gender stereotypes have set in during later childhood (Metz, 2007; Steele, 1997). Prior research suggests that children as young as four years old can successfully build and program simple robots while learning a range of engineering 
concepts in the process (Bers et al., 2002; Cejka et al., 2006; Perlman, 1976; Sullivan et al., 2013; Wyeth, 2008).

Robotics and computer programming in early childhood education can also foster the development of a range of cognitive and social skills. For example, early studies with the text-based programming language Logo have demonstrated that computer programming can help young children with number sense, language skills, and visual memory (Clements, 1999). Prior research has also shown that robotics can help children develop a stronger understanding of mathematical concepts such as number, size, and shape in much the same way that traditional materials like pattern blocks, beads, and balls do (Brosterman, 1997; Resnick et al., 1998). Unlike many other types of technology such as iPad apps and educational games, robotics activities do not involve sitting alone, in front of a screen. Rather, robotic manipulatives allow children to develop fine motor skills and hand-eye coordination while also engaging in collaboration and teamwork (Lee, Sullivan, \& Bers, 2013).

The study looks at very young children in kindergarten through second grade participating in a robotics and programing intervention at school. This research will determine whether introducing robotics and programming in early childhood allows both boys and girls to excel in the traditionally masculine areas of robotics and programming. Additionally, if gender differences in achievement are present, it will look at whether grade (kindergarten, first, or second) plays a role.

\section{Method}

\section{Research Questions}

This study examines newly forming attitudes and ideas young children have about technology and engineering products and young children's performance on robotics and programming tasks. Specifically, this study asks the following research questions:

1) Do young children have any pre-conceived notions or gender stereotypes about technology and engineering tools such as the KIWI robot?

2) Are there any gender differences in young children's mastery of KIWI robotics and programming concepts?

3) Does children's performance on KIWI robotics and programming concepts vary by grade level (Kindergarten, first, and second grade)?

\section{Sample}

$N=45$ children in Kindergarten through second grade participated in this research ( $n=18$ kindergarteners, $n=16$ first graders, $n=11$ second graders). Participating students attended an urban, public, early education school that serves children in Pre-Kindergarten through third grade. Massachusetts State demographic and census information reports that the school is $72 \%$ Hispanic, 69\% Limited English Proficiency, 65\% Free or Reduced Lunch, 15\% Special Education (Massachusetts Department of Elementary and Secondary Education, 2013).

\section{Procedure}

Participating children completed an eight-week robotics and programming curriculum in their classrooms taught by research assistants from the DevTech Research Group at Tufts University. Lessons were taught once a week and lasted approximately one hour. Trained research assistants taught the content to ensure consistency of curriculum implementation across classrooms; however, the children's regular classroom teachers were in the rooms to assist with behavioral management and small group work. 
Prior to the curriculum implementation, children participated in one-on-one interviews with the researchers to determine any knowledge, attitudes, or opinions they had about the KIWI robotics kit and various other common technology and engineering tools. Due to the young age of the participants, these interviews took a hands-on and play-based approach. For example, children were given toys, games, and kits to manipulate and explore to spark conversation and prompt discussion during the interview. Individual data on children's programming and robotics knowledge were collected one week after completion of the curriculum. All assessments were presented as games and activities rather than as formal tests.

\section{The KIWI Robotics Kita}

Children used the KIWI (Kids Invent With Imagination) robotics prototype because it was developed specifically for use with children ages four to seven years (see Figure 1). This prototype was developed by the DevTech Research Group at Tufts University through funding from the National Science Foundation and contains easy to connect robotic elements including three motors, a sound (clap) sensor, a distance sensor, a light sensor, and a light output (lantern). KIWI does not require any screen-time or even a computer to be programmed. Instead, its actions are programmed using CHERP, a tangible programming language consisting of interlocking wooden blocks (see Figure 1). Each CHERP block represents a different action the robot can carry out, such as spinning, moving forward, or beeping. The KIWI robot has an embedded scanner that allows users to scan barcodes on the CHERP blocks and send a program to the robot instantaneously.

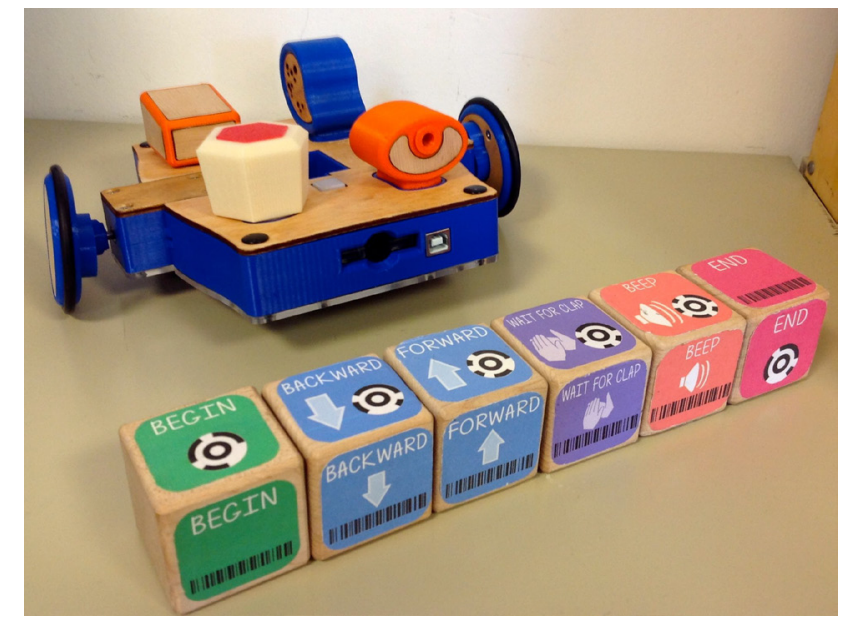

Figure 1 KIWI Robot and CHERP Programming Blocks

\section{The Robotics Curriculum}

The Positive Technological Development (PTD) framework developed by Bers (2012) was used to guide the development and implementation of the robotics curriculum in this study. The PTD framework guides the development, implementation, and evaluation of educational programs that use new technologies to promote learning as an aspect of positive youth development. PTD is a natural extension of the computer literacy and the technological fluency movements that have influenced the world of education but adds psychosocial and ethical components to the cognitive ones (Bers, 2008, 2012). From a theoretical perspective, PTD is an interdisciplinary approach that integrates ideas from the fields of computer-mediated communication, computer-supported collaborative learning, and the Constructionist theory of learning developed by Papert (1993), and views them in light of research in applied development science and positive youth development. 
As a theoretical framework, PTD proposes six positive behaviors (six C's) that should be supported by educational programs that use new educational technologies, such as KIBO, a robotics construction kit developed to teach programming and engineering to young children. These behaviors are creation, creativity, communication, collaboration, community building, and choices of conduct (Bers 2012). These "6 Cs" were used as a guide to create the robotics curriculum theme, lesson plans, and activities.

Aligned with the " $\mathrm{C}$ " of Community Building, the robotics lessons were completed over the course of eight weeks and were integrated with a larger curriculum unit called "Me and My Community" in which students explored their own identities, became connected to their school community, and learned about their neighborhoods. Each lesson was approximately one hour long and engaged children in a combination of hands-on building and programming challenges. In addition to the hands-on work with the robotics and programming tools, the robotics instructors used songs, games, picture books, and activities to reinforce the concepts being taught. For example, children learned the different parts of the KIWI robot with the "Robot Parts Song" (set to the tune of "Dry Bones") and mastered the programming commands by playing "Simon Says" with the CHERP programming blocks (See Table 1 for a sample class structure).

\section{Table 1: Sample Lesson Structure}

\section{Session 1. What is a Robot?}

Warm-Up Activity (approximately 15 minutes)

Discuss: What is a robot? Where can we find robots in the real-world?

Play: Jump for Robots (show a variety of pictures of robots and non-robots. Children jump if they think the picture is of a robot and stay still if it is not a robot. Discuss why.)

Introduce KIWI Parts: motors, main body, "brain" of the robot or microprocessor, wheels, and light-bulb.

Small-Group Activity (approximately 30 minutes)

Build your robot using the robot parts you just learned. Decorate using craft and recycled materials to represent you and the members of your group. Bring your robot to a "testing" station to get a program (children have not yet learned about programming) to see if it is sturdy and all parts are connected correctly.

Closing Activity (approximately 15 minutes)

Share: Share robotic creations. What was hard and difficult?

Sing: Sing \& dance The Robot Parts Song to reiterate the function of each KIWI robotic part and how they are connected.

Free-Play: Free play exploring robotic parts and the programming blocks (children will learn about the programming blocks during the structured activity in Lesson 2).

The programming and building challenges in each lesson encouraged children to use and expand upon concepts taught the previous week. During each class, there was also time provided for free play and exploration once children completed the given challenge. The last two weeks of the curriculum focused on the students' culminating project: an interactive robot map representing their community (see Figure 2). Each class mapped out the places in their community that were significant to them such as their homes, the school, churches, grocery stores, and restaurants. Once the maps were complete, the class worked in groups of 2-3 on building and programming robots to 
move along the map, stopping at locations of their choosing. These groups were generally mixed gender, with some all male and all female groups. Groupings were created by the classroom teacher and based primarily on students' ability to work effectively with one another. Each group worked at its own pace and created projects that were appropriately challenging for them. For example, some groups (particularly in second grade) used multiple sensors to stop at various locations in the community. Meanwhile, other groups just focused on creating simple programs to stop at one place on the map.

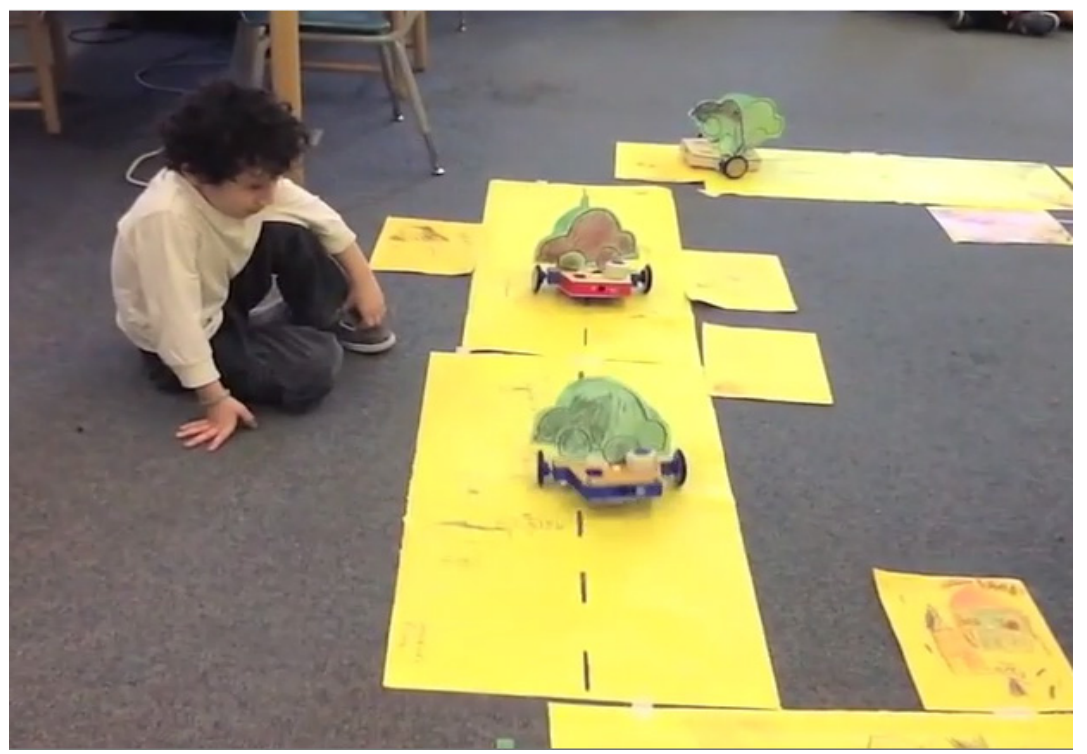

Figure 2: Community Map with KIWI Robots

Each of the " $6 \mathrm{Cs}$ " of the PTD framework was targeted through ongoing classroom practices with the robotics kit. This curriculum engages young learners in the following:

1. Content creation, by designing a KIWI robot and programming its behaviors.

2. Creativity, by making and programming personally meaningful projects and integrating different media such as robotics, motors, sensors, recyclable materials, arts and crafts, and a tangible programming language. Children's final "Me and My Community" projects involved designing and decorating maps and creating neighborhood structures.

3. Collaboration, by engaging children in a learning environment that promotes working in teams and sharing limited resources.

4. Communication, through engaging in small group conversations and planning sessions throughout the process of building and programming a KIWI robot. Additionally, each class began and ended with a "Technology Circle" discussion with the whole class sharing ideas and presenting their work.

5. Community-building, through opportunities to share their robotics work with one another in Technology Circle and their parents through teacher-curated open houses. By integrating with the "Me and My Community" theme, children also learned about the people, places, and important aspects of their greater neighborhood community.

6. Choices of conduct, through constant decision making about sharing materials, working with a partner or small group, and navigating social interactions. 


\section{Assessments}

Two quantitative assessments (the "Robot Parts Task" and the "Solve-Its Task") were used to measure children's robotics and programming knowledge after completing the curriculum and one qualitative assessment (the "Gender and Technology Attitudes Protocol") was used with a subset of participants to address young children's initial attitudes about robotics and technology. The "Robot Parts Task" was used to determine each child's robotics knowledge and the "SolveIt" assessment was used to measure each child's programming knowledge. Each of these assessments was completed on an individual basis. Because children worked in small groups during the curricular activities, it was important to implement assessments as individual tasks to see what types of challenges children could solve on their own.

Both the Robot Parts Task and the Solve-Its assessment were administered as post-tests, and no content knowledge pre-tests were administered. Both assessments addressed KIWI-specific content, meaning children would not be able to solve any of the tasks without prior knowledge of the kit. KIWI was a research prototype that was not commercially available at the time of this research. Because no children in the study had any prior exposure to KIWI, they all began the curriculum with the same baseline knowledge of KIWI robotics and programming.

The Technology Attitudes Protocol was administered to determine whether the young children in this study had any gender-based biases towards robotics and engineering tools. This interview was play-based and allowed children to use a variety of robotic tools (including the KIWI kit) prior to curriculum implementation and share their thoughts with a researcher. Since the participating children had no prior knowledge about KIWI, their initial attitudes were captured during the pretest when they were exposed to the robot for the first time.

\section{Gender and Technology Attitudes Protocol}

Qualitative data on children's attitudes about technology were collected using a hands-on Gender and Technology Attitudes Protocol, developed specifically for this study. This protocol was first tested with children in pre-kindergarten through second grade and it was determined that prekindergarten children had difficulty understanding this assessment and completing it in its entirety. Therefore children sat down with the researcher one-on-one at and played with a variety of technology and engineering tools including the following: Legos, the KIWI robotics kit, and a computer. They were asked to decide who from their class would enjoy playing with each item most: boys, girls, or both equally. The purpose of this line of questioning was to determine whether at this young age children see some tech tools as inherently masculine, feminine, or neither/both and to share their reasoning. Children were given time for hands-on play with the tools as they came up with their answers.

\section{Robots Parts Task}

The "Robot Parts task" was used to assess children's individual knowledge of the different KIWI robotic parts and their functions. This assessment was developed by the DevTech Research Group and based on in-class assignments that teachers and researchers designed to be used with young children during robotics classes. In order to complete this task, children sat down with researchers one on one and were asked to help the researcher identify different parts of the robot and their functions. For example, researchers asked, "what part should I use if I want my robot to turn its light on?" with the correct answer being the light output. After each question, children would pick up or point to the part they thought was correct and their answers were recorded. Children were asked to identify three sensors (light, distance, and sound), the light output, and the motors. Children received a point for correctly identifying each of these five components, yielding scores that could range from 0 (could not identify any of the parts) to 5 (correctly identified all parts). 


\section{Solve-Its}

"Solve It" tasks were used to assess children's individual programming knowledge at the end of curriculum implementation. The Solve-It tasks were developed to target areas of foundational programming ability (Strawhacker \& Bers, 2015; Strawhacker, Sullivan, \& Bers, 2013). This assessment is intended to test students' mastery of programming concepts, from basic sequencing through repeat loops. The Solve-It tasks require children to listen to stories about a robot and then spend 3-5 minutes attempting to create the robot's program using CHERP programming icons on paper (See Figure 3). For example, one story was about the bus from the children's song "Wheels on the Bus" (Strawhacker \& Bers, 2015; Strawhacker et al. 2013,).

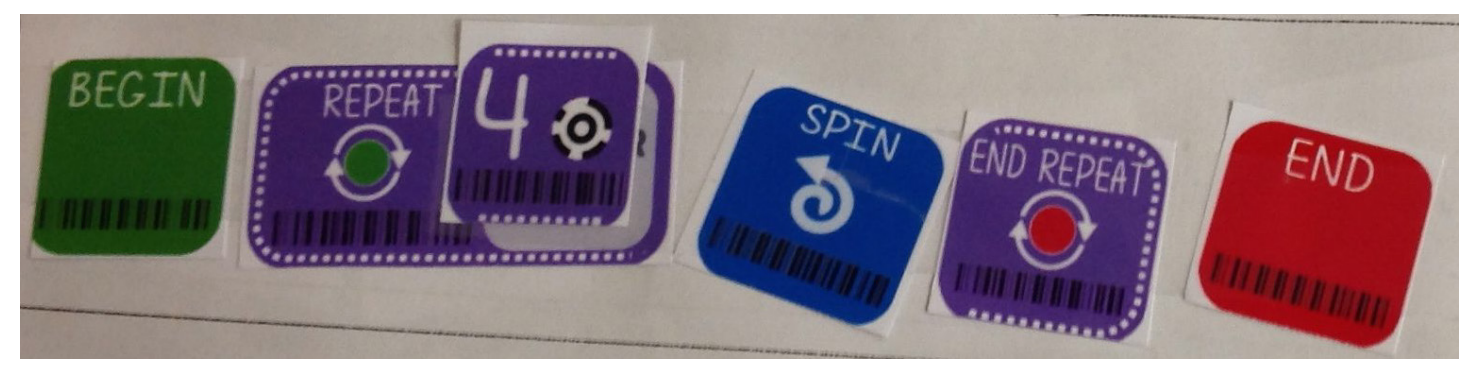

Figure 3: Sample Child-Completed "Wheels on the Bus" Solve-It

Seven Solve-Its were administered to the children in this study upon completion of the curriculum (see Table 2). The seven Solve Its tested the following programming concepts: Easy Sequencing, Hard Sequencing, Sequencing with the "Wait-For" Command, Easy Repeat Loops with Number Parameters, Hard Repeat Loops with Number Parameters, Easy Repeat Loops with Sensor Parameters, and Hard Repeat Loops with Sensor Parameters. Tasks increased with difficulty, with Sequencing being the simplest to answer and Repeat Loops with Sensor parameters being the most difficult. Within each type of task, there were both "easy" or "hard" based challenges. The label of "easy" versus "hard" was determined based on how many commands children needed to sequence (i.e., easy tasks had fewer blocks for children to sequence than hard tasks, but both addressed the same programming concept). See Table 2 for a more detailed description of each Solve-It task and the difficulty level of each task.

Each of the Solve-It tasks described above was scored on a 0-6 rubric based on how close the child's program came to being completely correct (a score of 6). The score of 0-6 was derived from sub-scores targeting concepts of control flow and action sequencing. In the original development of this scoring mechanism, inter-scorer reliability tests for the Solve-Its assessment showed precise agreement (two items; $K=0.902$, p < 0.001) (Strawhacker $\&$ Bers, 2015). In the present study, one trained researcher from the original study scored all Solve-Its.

Table 2: Solve-It Tasks

\begin{tabular}{lll}
\hline $\begin{array}{l}\text { Solve-It } \\
\text { (organized by } \\
\text { difficulty) }\end{array}$ & Solve-It Name & Concepts Targeted \\
\hline 1 & Easy Sequencing & $\begin{array}{l}\text { Sequencing and order of action blocks (4 commands } \\
\text { total) } \\
\text { "Begin" and "End" blocks }\end{array}$ \\
\hline 2 & Hard Sequencing & $\begin{array}{l}\text { Sequencing and order of action blocks (6 commands } \\
\text { total) } \\
\text { "Begin" and "End" blocks }\end{array}$ \\
\hline
\end{tabular}




\begin{tabular}{|c|c|c|}
\hline $\begin{array}{l}\text { Solve-It } \\
\text { (organized by } \\
\text { difficulty) }\end{array}$ & Solve-It Name & Concepts Targeted \\
\hline 3 & "Wait-For" Command & $\begin{array}{l}\text { "Wait-for clap" block to program sound sensor } \\
\text { Basic Sequencing ( } 4 \text { commands) }\end{array}$ \\
\hline 4 & $\begin{array}{l}\text { Easy Repeats with } \\
\text { Numbers }\end{array}$ & $\begin{array}{l}\text { "Repeat" and "End Repeat" blocks to create a Repeat } \\
\text { Loop } \\
\text { Number parameters to modify Repeat Loop } \\
\text { Sequencing } 1 \text { action command with the Repeat com- } \\
\text { mands ( } 5 \text { total) }\end{array}$ \\
\hline 5 & $\begin{array}{l}\text { Hard Repeats with } \\
\text { Numbers }\end{array}$ & $\begin{array}{l}\text { "Repeat" and "End Repeat" blocks to create a Repeat } \\
\text { Loop } \\
\text { Number parameters to modify the Repeat Loop } \\
\text { Sequencing } 2 \text { action commands with the Repeat com- } \\
\text { mands ( } 6 \text { total) }\end{array}$ \\
\hline 6 & $\begin{array}{l}\text { Easy Repeats with } \\
\text { Sensor Parameters }\end{array}$ & $\begin{array}{l}\text { "Repeat" and "End Repeat" blocks to create a Repeat } \\
\text { Loop } \\
\text { Sensor parameters to modify the Repeat Loop } \\
\text { Sequencing } 1 \text { action command with the Repeat com- } \\
\text { mands ( } 5 \text { total) }\end{array}$ \\
\hline 7 & $\begin{array}{l}\text { Hard Repeats with } \\
\text { Sensor Parameters }\end{array}$ & $\begin{array}{l}\text { "Repeat" and "End Repeat" blocks } \\
\text { Sensor parameters to modify Repeat Loop } \\
\text { Sequencing } 2 \text { action commands with the Repeat com- } \\
\text { mands ( } 6 \text { total) }\end{array}$ \\
\hline
\end{tabular}

Note. Solve it tasks are numbered based on overall difficulty of concept. For example, both the "easy" and "hard" sequencing tasks (tasks 1 and 2) are typically easier for children than the easiest "Repeats" task (task 4). Within each category, there may be easy and hard tasks (for example, and "easy sequencing" and a "hard sequencing" task). Both target the same conceptual understanding, but the more difficult task has more actions to sequence.

\section{Results}

\section{Gender and Technology Attitudes Protocol}

$N=42$ children in Kindergarten through second grade completed the Gender and Technology Attitudes protocol. This was administered before any curriculum intervention to address any preconceived notions the children have about different technology and engineering tools. Children were shown Legos, a KIWI robot, and a computer.

Of all of the tools, the legos evoked the most prominent gender-based attitudes from participants. Out of the 42 children more than half (64\%) said that boys would enjoy playing with the legos more. Children were prompted to elaborate on their reasoning. A common theme that came up was the color of the legos (a mix of primary colors including yellow, red, and blue). For example, one child stated, "girl legos are pink." Other children commented that boys enjoy building more 
than girls. For example, one child stated, "boys would build something with legos. Girls don't play with legos" (See Table 3 for more examples).

When it came to the computer, more than half of the participants (62\%) stated that both boys and girls would like the computer. Children made comments like, "boys and girls both like playing on the computer." Still, some children mentioned that while both boys and girls enjoy the computer, they would engage in different activities when using it. For example, one child stated: "they [boys and girls] could both play games on computer. But they like different games." Another child elaborated by saying, "boys play games like Batman. A girl computer would have girl games."

Table 3: Gender \& Technology Attitudes Significant Quotes

\begin{tabular}{|l|l|}
\hline \multicolumn{1}{|c|}{ Technology } & \multicolumn{1}{c|}{ Significant Quotes } \\
\hline Legos & $\begin{array}{l}\text { "These aren't girl legos." } \\
\text { "Girl legos are pink." } \\
\text { "The boys would build something. Girls don't play with legos." }\end{array}$ \\
\hline KIWI & $\begin{array}{l}\text { "Because it's a lot blue. If a different color girls would like it." } \\
\text { "It looks kinda cool and it looks all blue. There's no girl colors." } \\
\text { "Boys like the blue and things that are fast." } \\
\text { "Boys like to take those things [the sensor and output modules and motors] } \\
\text { apart" }\end{array}$ \\
\hline Computer & $\begin{array}{l}\text { "Boys play games like Batman. A girl computer would have girl games } \\
\text { and be pink." } \\
\text { "They could both play games on computer. They like different games." } \\
\text { "Boys and girls both like to play on the computer" }\end{array}$ \\
\hline
\end{tabular}

When it came to the KIWI robot, more than a quarter of the children (33\%) believed that boys would enjoy playing with KIWI more. Just like with the legos, the color of the KIWI robot was one of the common themes that emerged. The KIWI prototypes used in this study were blue and tan/wood in color. Children made comments such as "there's no girl colors" and "because it's a lot blue. If a different color girls would like it." Children also had opinions on the lights and sounds associated with the different robotic modules. For example, on child stated, "Those sounds are gonna bother some girls" about the beeps and noises the robot made. Despite this, about half of the children (53\%) stated that both boys and girls would like KIWI. Some children could not articulate why both boys and girls would like KIWI, simply explaining that "its cool" and they like it. Others said the shapes and the toy-like feel would appeal to girls.

\section{Robot Parts Task}

$N=41$ children completed the Robot Parts task to test their knowledge of the parts and functions of the KIWI robot. The task was scored out of a possible five points and mean scores were calculated by grade and by gender. Results show that, on average, all grades and genders performed very well on this assessment, with an overall mean score of 4.32 on this task $(S D=.986)$.

Next, a two-way Analysis of Variance (ANOVA) was used to determine if gender, grade level, or the interaction of grade and gender had any significant effect of children's performance on the Robot Parts Task. Analysis revealed that there was no significant main effect for gender and no 
significant effect for the interaction of grade and gender ( $p>.05)$, suggesting that boys and girls performed equally well on this assessment. However, there was a significant main effect for grade level $F(2,35)=3.637, p<.05$. This indicates that at least two grades performed significantly differently from one another. Post hoc Tukey HSD testing was used to determine where these differences lie. Results show that first grade $(M=4.5, S D=.941)$ and second grade $(M=4.9, S D=.316)$ both performed significantly better than the kindergarteners $(M=3.82 S D=1.07, \mathrm{p}<.05)$. However, first and second grade did not perform significantly differently from one another $(\mathrm{p}>.05)$.

\section{Solve-Its}

Children were administered seven Solve-It tasks after completing the curriculum that targeted various programming concepts. Each task increases in difficulty as the Solve-It number increases (i.e. Solve-It 1 is the simplest task while Solve-It 7 is the most complex task). Each task was scored out of a possible six points. Descriptive results show that, on average, children performed better on the first three tasks related to the basic sequencing than on the more challenging concepts such as repeat loops and sensor parameters. As the Solve-Its increased in difficulty, mean scores tended to decrease, for all grades and genders (see Figures 4 and 5).

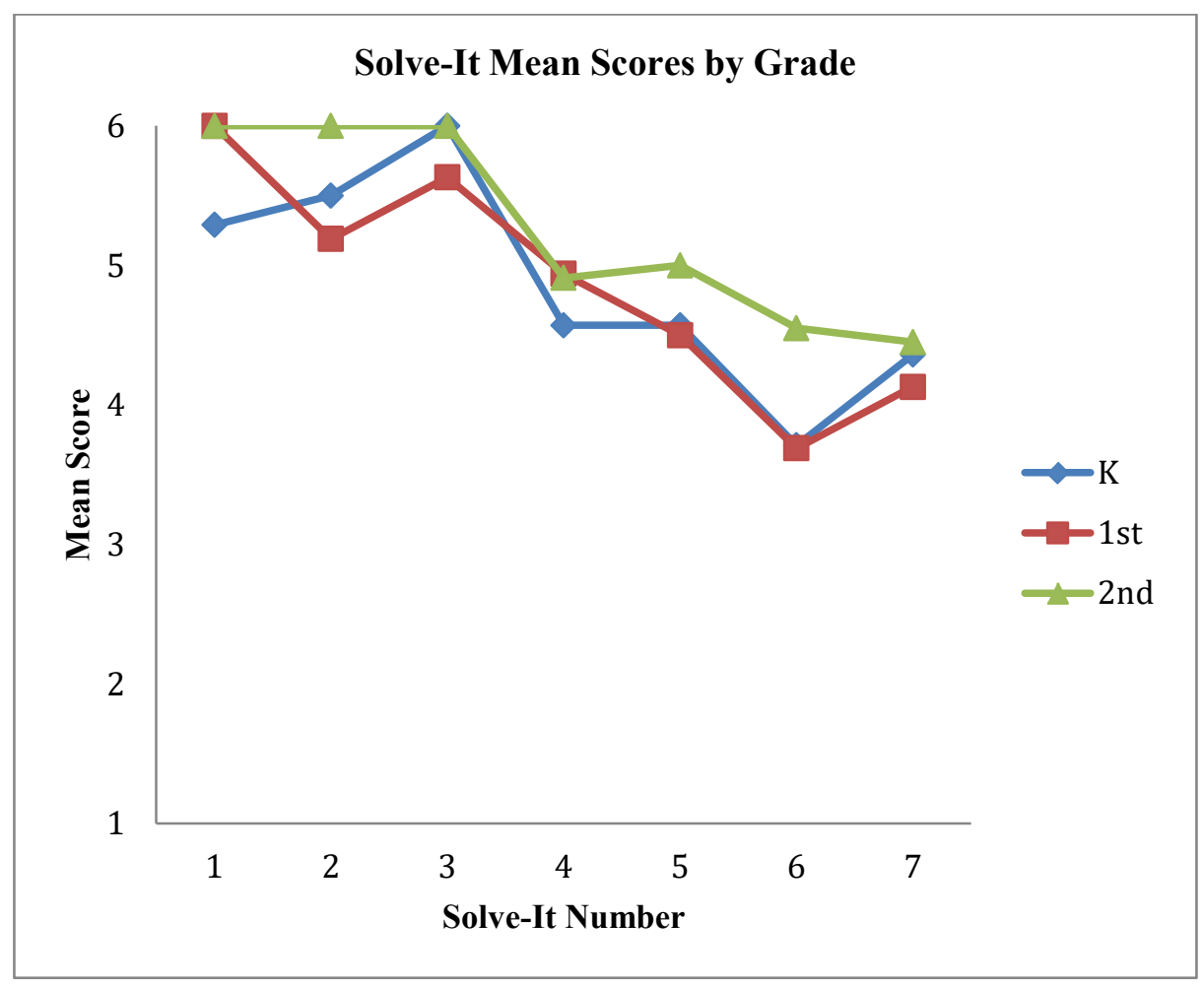

Figure 4: Solve-It Mean Scores by Grade 


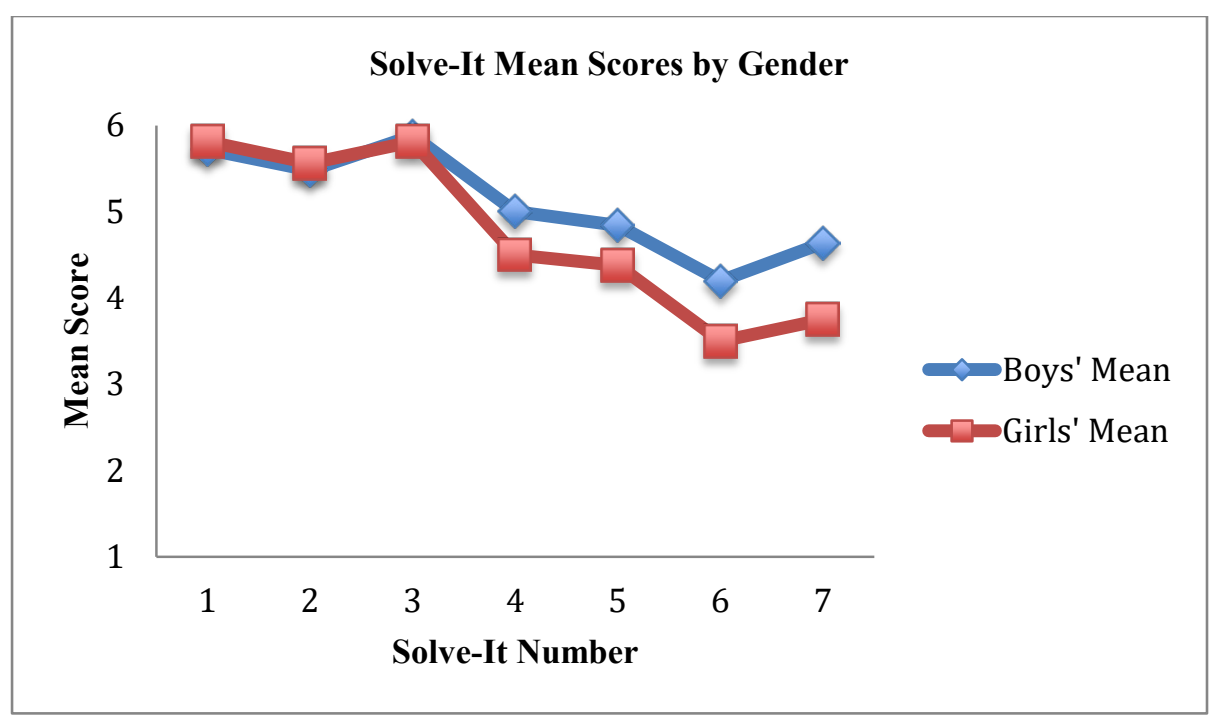

Figure 5: Solve-It Mean Scores by Gender

Next, a two-way Analysis of Variance (ANOVA) was carried out for each of the seven Solve-It tasks to determine if gender (male or female) or grade level (kindergarten, 1st, 2nd) had a significant effect on children's performance on each of the Solve-It tasks. Each Solve-It task assessed a different programming concept taught within the KIWI robotics curriculum and as such, was analyzed separately from one another. The two-way ANOVA was also used to see if the interaction of grade and gender had any effect on Solve-It performance. For example, this test was used to see if the effect of gender was influenced by grade level.

On the first four Solve-It tasks, that target basic programming skills including concepts of sequencing, simple repeat loops, and use of the "wait for" block, two-way ANOVAs found no significant effects for grade, gender, or the interaction of grade and gender $(p>.05)$. This indicates that all members of the sample, regardless of gender or grade level, displayed equal mastery of these basic concepts.

However, significant effects were found on all of the advanced Solve-It tasks (See Figure 6). Beginning with the "Hard Repeat Loops with Numbers" task, there was a significant main effect for gender $F(1,35)=4.305, p<.05$, indicating that the mean score for boys $(M=4.84, S D=1.03)$ was significantly greater on this task that the mean score for girls $(M=4.38, S D=.619)$. There was no main effect on these tasks for grade level or the interaction of grade and gender found $(\mathrm{p}>.05)$.

On both "Repeat Loops with Sensors" tasks (Solve-It numbers 6 and 7) significant effects were found. On the Easy Repeats with Sensors task, there was a significant main effect for gender $F(1$, $35)=10.197, p<.005$ and a significant main effect for grade level $F(2,35)=4.965, p<.05$.

However, there was no significant interaction effect for grade level and gender together ( $>$.05). This indicates that the mean score for boys $(M=4.20, S D=1.23)$ was significantly greater on this task than the mean score for girls $(M=3.50, S D=.516)$. Post-hoc Tukey HSD testing for grade level showed that on the Easy Repeats task the second graders performed significantly better than the first graders $(p<.05)$ but there were no other significant grade level differences.

On the Hard Repeats with Sensors task there was a significant main effect for gender $F(1,35)=$ $12.120, p<.005$, indicating that the mean score for boys $(M=4.64 S D=.810)$ was significantly greater on this task that the mean score for girls $(M=3.75, S D=.856)$. There was no main effect for grade level or the interaction of grade and gender found $(\mathrm{p}>.05)$. 


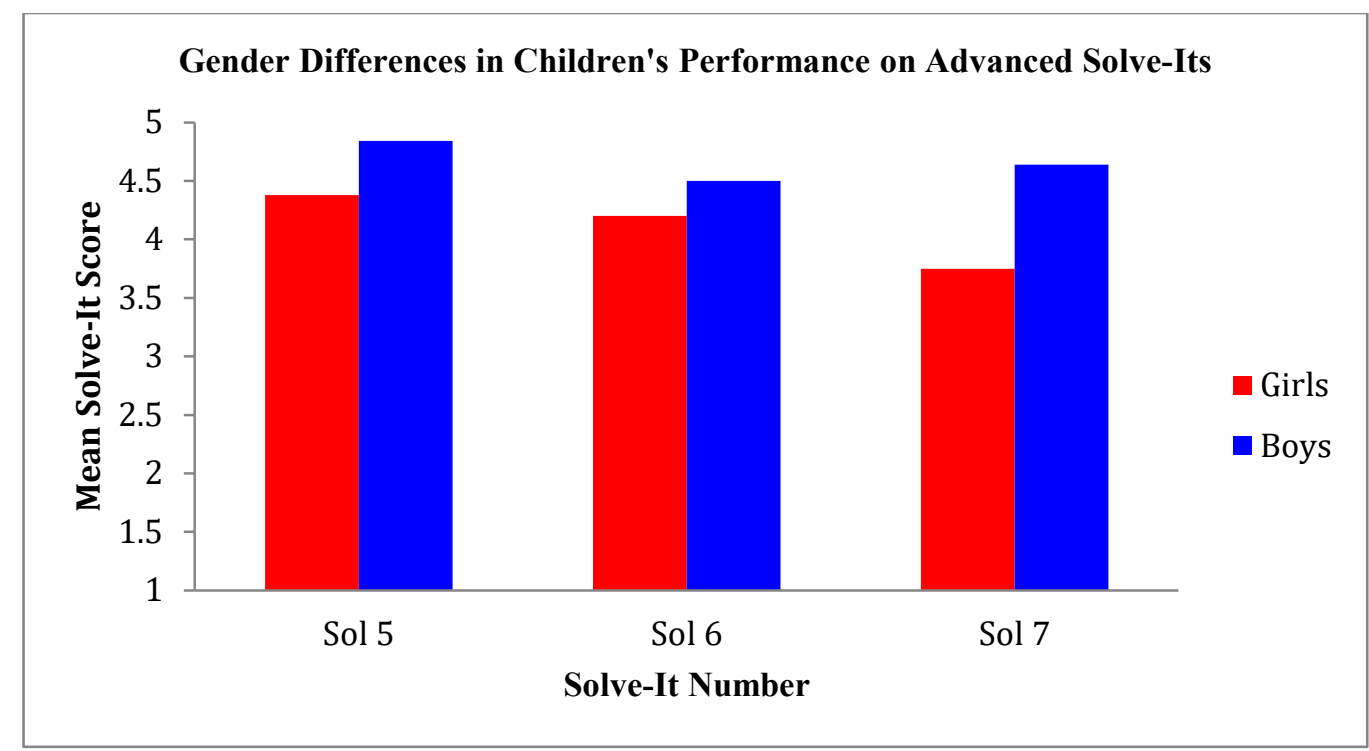

Figure 6: Gender Differences in Children's Performance on Advanced Solve It Tasks

Note. Sol 5 refers to the Hard Repeat Loops with Number Parameters task, Sol 6 refers to Easy Repeats with Sensor Parameters, and Sol 7 refers to Hard Repeats with Sensor Parameters.

\section{Discussion}

\section{Initial Attitudes toward Technology}

The Gender and Technology Attitudes pretest uncovered interesting qualitative findings on children's initial attitudes about technology and engineering in their early childhood years. Legos evoked the strongest gender based attitudes, with the majority of children explaining that boys would like building with legos more than girls. This strong categorization of "girl" and "boy" toys is not surprising given prior research that children begin to stereotype opinions about toys and gender beginning in preschool (Martin, Eisenbud, \& Rose, 1995). Children used their own observations from classmates and family members to help them make this stereotypic categorization. For example, one student mentioned that, "almost all the boys in my classroom go and play legos" and another stated that, "girls in our class don't like using lego." Children also relied on their knowledge of the packaging and marketing of legos. They mentioned things like "boy legos" vs. "girl legos." Finally, several students also mentioned the word "building" and often stated that boys enjoy building activities more than girls.

Children showed milder gender attitudes towards the electronic technologies they were shown. The majority of children stated that both boys and girls would equally enjoy using the computer and the KIWI robot. For example, many children explained that all kids love robots. This may because the KIWI robot was so novel to the participants that they were unable to use stereotypes to categorize it in the same way as the legos (something with which they were all very familiar and had possibly received marketing exposure). Despite this, many children still commented a great deal on the aesthetic design of the KIWI robot and why this design might be more appealing to boys. Several children commented on the prominent blue color featured on the robot and the fact that it looks like a car when fully assembled. This information was used by the KIWI design team to change the look and feel of the KIWI robot in later iterations so that it would be visually appealing to both boys and girls (Sullivan, Elkin, \& Bers, 2015). 


\section{Mastery of Robotics Knowledge}

When it came to children's mastery of knowledge, the Robot Parts Task demonstrated that the first and second graders performed significantly better than the kindergarteners. This task did not require any physical manipulation of the robot parts (children simply pointed to identify different pieces and their functions) so this grade level difference cannot be attributed to more developed fine motor skills on the part of the older children. It possible that the older children were more familiar with the sensors because many first and second graders in this study chose to use multiple sensors in their final projects. Meanwhile, the kindergarteners typically used just the sound sensor (or no sensor at all) in their final projects because this was easier to program. Despite this grade level difference, all three grades performed very well on the assessment, displaying a high level of mastery of the KIWI robot parts and functions.

Results from the Robot Parts Task also show that there were no significant differences found based on gender (male or female). The lack of gender differences found on this assessment is surprising because prior research by Sullivan and Bers (2013) using the LEGO RCX robotics kit and a similar tangible programming language found that kindergarten boys performed significantly better than girls at correctly building with robotic materials. This may be due to the fact that the assessment used in this study asked children to identify different robotic parts rather than actually build with them as in the prior study. Alternatively, it may provide initial evidence that the robotics materials found in the KIWI robotics kit may be more accessible to girls than the LEGO RCX kit. This is consistent with pretest findings from the Gender and Technology Attitudes protocol. In these pretesting interviews, the majority of children felt that boys enjoy using legos more than girls. Since the KIWI robotic kit did not require the use of any legos (unlike the prior study by Sullivan and Bers, 2013) this may explain why girls were more successful building with KIWI. Future research should look at comparing boys' and girls' ability to identify KIWI robotics parts as well as to physically build with them in order to provide stronger evidence that the KIWI kit is more accessible to girls than traditional commercially available products like LEGO.

Finding that boys and girls displayed equal mastery of KIWI robotics concepts suggests that, when developmentally appropriate tools are used, robotics can be an effective way to introduce young girls to the technology and engineering components of STEM. However, it is possible that it was not only the KIWI materials that allowed girls to succeed, but the nature of the curriculum and the collaborative learning environment that was fostered. In the curriculum implemented here, children worked in groups of 2-3 on all challenges. None of the challenges culminated in a competition, as is the case with many other popular robotics programs for elementary aged students such as the FIRST LEGO League. In the average FIRST competition team, boys outnumber girls 5 to 2 (Center for Youth and Communities, 2013). This is aligned with prior research demonstrating that for a variety of neural and chemical reasons boys are naturally more aggressive and competitive than girls (Gurian, 1996). Gneezy and Rustichini (2004) found that competition actually improves performance for boys but not for girls. Therefore, the collaborative nature of the curriculum implemented in this study, rooted in Bers (2012) Positive Technological Development framework, may be more of an appealing way for girls to learn robotics. Future research should look at the role curriculum design and group work in the success of boys' and girls' mastery of robotics concepts.

\section{Mastery of Programming Knowledge}

When it came to programming, boys and girls in this study performed equally well on all of the basic programming tasks such as sequencing a simple program in the correct order, using the "wait for" command, and sequencing a short repeat loop program using number parameters. This indicates that both boys and girls displayed equal mastery for of all of the basic concepts that are 
foundational to computer programming. This is contrary to prior research with older students that girls are more likely to struggle with programming than boys (Nourbakhsh et al., 2004).

Looking at the more advanced concepts such as programming repeat loops with sensors, boys performed significantly better than girls in many of the same ways highlighted by prior research on older students. There is not much research on gender differences in young children's performance on robotics and programming tasks, however, Nourbakhsh et al. (2004) looked at gender differences in high school robotics students. These authors found that girls were more likely to have struggled with programming than boys. Nourbakhsh et al. (2004) also found that girls entered the course with less confidence than boys. It is possible that these types of gender differences previously found in older girls and boys begin to emerge much earlier than previously assumed.

The advanced programming tasks administered draw heavily on basic math concepts such as number sense, counting, and problem solving. Prior research has shown that boys and girls solve math problems by employing different strategies. For example, girls tend to use more modeling or counting strategies, while boys tended to use more abstract strategies such as abstractly coming up with a solution derived from facts that they already know (Fennema, Carpenter, Jacobs, Franke, \& Levi, 2014). It is possible that using concrete strategies that girls are more likely to use, such as counting and observation, slowed them down when completing the timed Solve-It tasks. Children were given a maximum of only five minutes to complete each Solve-It task. Perhaps if more time were given (or the Solve-Its activities were given as a curricular project rather than a timed task) the results would have been different. Future work should delve into the types of mistakes girls and boys made programming during both projects and timed tasks to better develop curricular activities that match both styles of problem solving. Finally, it is important to note that although boys performed significantly better than girls on three solve-it programming tasks, girls still performed well on this task with mean scores of 3.75 or higher (out of 6 ) on all programming tasks completed.

Another interesting finding was the limited significant main effects for grade level and the interaction effects between grade level and gender. Only one Solve-It task, the simple Repeat Loops task, showed a significant main effect for grade level and revealed than the second graders performed significantly better than the first graders. Because the tasks rely heavily on math, problem solving, and working memory, it is surprising that the older students did not outperform the younger students on many more of the tasks. This provides some evidence that, regardless of grade, the programming language used was generally accessible to all early childhood students. However, this finding may also be attributed to the small sample size and, in particular, the small number of students representing second grade in this sample. These results should be interpreted cautiously until replications with a larger sample are performed.

Additionally, there were no significant interaction effects between grade and gender. Prior research has discussed that as children get older, gender stereotypes become more firmly ingrained and that these stereotypes can affect performance on assessments (Metz, 2007; Steele, 1997). However, in this study, there was no significant interaction between grade and gender on any of the programming or robotics tasks. This means that older children in this study were not more likely to display gender differences in their performance on the Robot Parts task or the Solve-It tasks than the younger children. One reason may be that the children in this study were all relatively young and similar in age (5-8 years). It is possible that if this study were larger and spanned more grades we would be able to see an interaction between grade and gender effects. 


\section{Limitations and Future Work}

The qualitative data in this study presented a preliminary look at the types of attitudes and opinions young children are beginning to form about which types of technology and engineering activities are appropriate and engaging for boys and girls. This study was the first piloting of the Gender and Technology Attitudes protocol. Future work will expand on this protocol to include a wider variety of technologies, in particular, other commercially available robotics kits. Additionally, the protocol will be expanded to prompt more open-ended conversations with children and hear more about their experiences with each tool being shown. Additionally, analysis for this study was greatly limited by a small sample size. Future work will attempt to further explore these ideas with a larger sample of children. Finally, in future work the researchers will administer both pre and post interviews to see if opinions change due to participation in a robotics curriculum.

Despite these limitations, several themes emerged that can be useful to educators and designers of technologies for children. For example, KIWI has gone through several different colors and aesthetic changes since the collection of this data (Sullivan et al., 2015). Based on that feedback, the KIWI robot kit was re-designed and is now commercially available as the KIBO robot (Sullivan et al., 2015). Similar follow-up interviews were done to determine a color that was popular amongst both boys and girls, resulting in a neutral orange KIBO robot. Additional arts and crafts platforms were also added to modify the "car-like" features and make the robot equally appealing to both boys and girls and suitable for creative play and design (See Figure 7) (Sullivan et al., 2015). Future research may further examine the impact of color preference and gender attitudes about technology.

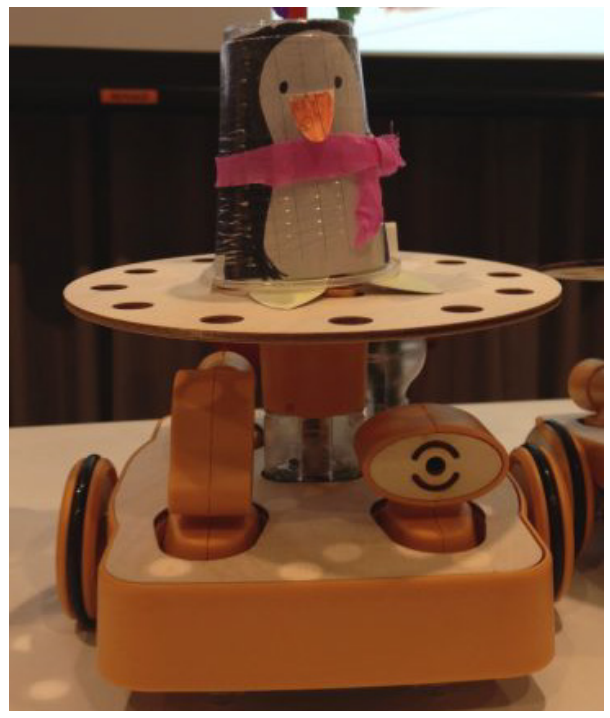

Figure 7: New KIBO robot with updated color and new motorized art platforms for personalizing. It is shown here decorated with arts and crafts to represent a penguin.

This study provided some evidence that young children are already forming gender-based opinions about technology and engineering beginning in kindergarten. It is possible that even younger children (in preschool and earlier) may have had different reactions to the technologies being shown in this study. Future work should explore the attitudes of younger children who may have less developed gender attitudes about technology.

The quantitative data collected in this study was limited by the typical constraints associated with educational research in public school settings. For example, throughout the curriculum implementation it was common for children to miss classes due to absences, behavioral difficulties, and to 
receive special services (i.e., special reading or math tutoring). While researchers attempted to keep track of these absences, attendance rosters were not always accurate because children often missed partial classes for unpredictable reasons. These same issues also prevented some students from completing the post-testing assessments, further limiting the sample size for this study.

Data analysis in this study was greatly limited by the relatively small sample size and uneven proportion of students in each group. Because of this, not all assumptions for the ANOVA were met. For example, normal distributions were not found for all of the Solve-It tasks, most likely due to the small sample. While we found no gender differences in knowledge for the basic solveit tasks, the small sample size may have influenced our ability to detect differences. The small sample size would mean an uneven distribution of groups may play a role in the discovery of main effects with ANOVA. Therefore, findings should not be generalized beyond this pilot sample and should be interpreted with caution and used to help inform the design of larger studies. Future work should replicate this study with a larger sample of children for more generalizable results. Additionally, both female and male research assistants helped with curriculum and assessment implementation. Due to personnel limitations, we were unable to control for the gender of robotics instructors. Future work should carefully control for the gender of the robotics instructors to determine if this has any impact on how children identify with the material being taught and their performance on tasks.

This study implemented a collaborative robotics curriculum that was very different from many of the widespread robotics competitions and clubs that are available nationwide for older children. It demonstrated an example of applying the Positive Technological Development framework in the context of early childhood robotics in which children mastered a variety of robotics and programming concepts. Future research should continue to examine this collaborative approach to robotics education as well as a variety of other teaching methods in order to determine the most effective way to engage young in engineering. Future work may also want to look at children's confidence in their abilities to see if confidence plays a role on performance on tasks and to determine the best curricular practices for instilling confidence.

\section{Conclusion}

Results from this study demonstrate that young children ages 4-7 are already beginning to decide which technology and engineering activities and materials are better suited to boys or girls. This is a pivotal time to introduce children to innovative new technologies, such as robotics, before they form gender-biased opinions of these tools.

Both boys and girls demonstrated a high level of success in the Ready for Robotics program. Girls and boys performed equally well on their knowledge of the functions of different components of the KIWI robotics kit, which was not the case for prior research with young children using Lego based robot kits. This provides preliminary evidence that the creation of new tools such as KIWI that do not look or feel like traditional engineering materials may trigger less gender stereotyping and allow both boys and girls to be engaged by them. This also suggests that the collaborative nature of the curriculum implemented in this study may be more successful at engaging girls than other traditionally competitive robotics programs. Although the boys in this study performed significantly better than girls on the advanced programming tasks, they performed equally well on all other tasks. Future research should continue to examine why boys performed better on these tasks so that this divide can be addressed.

The Ready For Robotics project allowed girls and boys to experience an engaging introduction to robotics and computer programming beginning in kindergarten. This program attempted to reach girls prior to the ingraining of extreme gender stereotypes; however, despite the young age of the participants in this sample, some gender differences were still present. As men continue to out- 
number women in fields like Computer Science and Engineering, further research examining the early experiences of young children with technology long before high school and college years is of growing importance.

\section{References}

Aschbacher, P. R., Li, E., \& Roth, E. J. (2010). Is science me? High school students' identities, participation and aspirations in science, engineering, and medicine. Journal of Research in Science Teaching, $47(5), 564-582$.

Bers, M. U. (2008). Blocks, robots and computers: Learning about technology in early childhood. New York: Teacher's College Press.

Bers, M. U. (2012). Designing digital experiences for positive youth development: From playpen to playground. Cary, NC: Oxford.

Bers, M. U., Ponte, I., Juelich, K., Viera, A., \& Schenker, J. (2002). Teachers as designers: Integrating robotics in early childhood education. Information Technology in Childhood Education, 1, 123-145.

Brickhouse, N. \& Potter, J. (2001). Young women's scientific identity formation in an urban context. Journal of Research in Science Teaching, 38(8). 965-980.

Brosterman, N. (1997). Inventing kindergarten. New York: H.N. Abrams.

Cejka, E., Rogers, C., \& Portsmore, M. (2006). Kindergarten robotics: Using robotics to motivate math, science, and engineering literacy in elementary school. International Journal of Engineering Education, 22(4), 711-722.

Center for Youth and Communities at Brandeis University. (2013). Executive summary - Evaluation of the FIRST LEGO league "Senior Solutions" Season (2012-13). Retrieved from: http://www.firstinspires.org/sites/default/files/uploads/resource_library/impact/impact-executivesummary-first-lego-league-impact-study-2012-2013.pdf

Clements, D. H. (1999). Young children and technology. In Dialogue on early childhood science, mathematics, and technology education. Washington, DC: American Association for the Advancement of Science.

Fennema, E.; Carpenter, T., Jacobs, V.; Franke, M.; \& Levi, L. (2014). A longitudinal study of gender differences in young children's mathematical thinking. Educational Researcher, 27(5), 6-11.

Gneezy, U., \& Rustichini, A. (2004). Gender and competition at a young age. The American Economic Review. 94(2), 377-381.

Gurian, M. (1996). The wonder of boys. New York: Tarcher/Putnam.

Hill, C., Corbett, C., \& St. Rose, A. (2010). Why so few women in science, technology, engineering, and mathematics. Washington, DC: Washington, DC: American Association of University Women; 2010. Retrieved from http://www.aauw.org/learn/research/whysofew.cfm

Hughes, R. M.; Nzekwe, B., \& Molyneaux, K. J. (2013). The single sex debate for girls in science: A comparison between two informal science programs on middle school students' STEM identity formation, Research In Science Education, 43(5).

Lee, K., Sullivan, A., \& Bers, M. U. (2013). Collaboration by design: Using robotics to foster social interaction in Kindergarten. Computers in the Schools, 30(3), 271-281.

Madill, H., Campbell, R.G., Cullen, D. M., Armour, M. A., Einsiedel, A. A., Ciccocioppo, A. L.,...Coffin, W. L. ( 2007). Developing career commitment in STEM-related fields: Myth versus reality. In R. J. Burke, M. C. Mattis, \& E. Elgar (Eds.), Women and minorities in science, technology, engineering and mathematics: Upping the numbers (pp. 210-244). Northhampton, MA: Edward Elgar Publishing.

Markert, L. R. (1996). Gender related to success in science and technology. The Journal of Technology Studies, 22(2), 21-29. 
Martin, C. L., Eisenbud, L., \& Rose, H. (1995). Children's gender-based reasoning about toys. Child Development, 66, 1453-1471.

Martin, C. L., \& Ruble, D. N. (2004). Children's search for gender cues: Cognitive perspectives on gender development. Current Directions in Psychological Science, 13, 67-70.

Massachusetts Department of Education. (2013). School profiles. Retrieved from: http://profiles.doe.mass.edu/

Metz, S. S. (2007). Attracting the engineering of 2020 today. In R. J. Burke, M. C. Mattis, \& E. Elgar (Eds.), Women and minorities in science, technology, engineering and mathematics: Upping the numbers (pp. 184-209). Northampton, MA: Edward Elgar Publishing.

Milto, E., Rogers, C., \& Portsmore, E. (2002). Gender differences in confidence levels, group interactions, and feelings about competition in an introductory robotics course.In ASEE/IEEE Frontiers in Education Conference.

National Center for Women and Technology. (2011). Women and information technology by the numbers. Fact sheet. Retrieved December 5, 2011 from http://www.ncwit.org/pdf/BytheNumbers09.pdf

Nourbakhsh, I., Hammer, E., Crowley, K., \& Wilkinson, K. (2004). Formal measures of learning in a secondary school mobile robotics contest. In IEEE International Conference on Robotics and Automation (ICRA)

Papert, S. (1993). The children's machine: Rethinking school in the age of the computer. New York: Basic Books.

Perlman, R. (1976). Using computer technology to provide a creative learning environment for preschool children. Logo memo no 24, Cambridge, MA: MIT Artificial Intelligence Laboratory Publications 260.

Resnick, M., Martin, F., Berg, R., Borovoy, R., Colella, V., Kramer, K., \& Silverman, B. (1998). Digital manipulatives. Proceedings of the CHI '98 conference, Los Angeles, April 1998.

Shapiro, J., \& Williams, A. (2011). The role of stereotype threats in undermining girls' and women's performance and interest in STEM fields. Sex Roles, 66(3-4), 175-183. Springer Science+Business Media, LLC.

Spencer, S., Steele, C., \& Quinn, D. (1999). Stereotype threat and women's math performance. Journal of Experimental Social Psychology, 35, 4-28.

Steele, C. M. (1997). A threat in the air: How stereotypes shape intellectual identity and performance. American Psychologist, 52, 613-629.

Strawhacker, A. L., \& Bers, M. U. (2015). "I want my robot to look for food": Comparing children's programming comprehension using tangible, graphical, and hybrid user interfaces. International Journal of Technology and Design Education, 25(3), 293-319.

Strawhacker, A. Sullivan, A. \& Bers, M. U. (2013). TUI, GUI, HUI: Is a bimodal interface truly worth the sum of its parts? In Proceedings of the 12th International Conference on Interaction Design and Children (IDC '13). ACM, New York, NY, USA, 309-312. Retrieved from https://ase.tufts.edu/DevTech/publications/p309-strawhacker.pdf

Sullivan, A., \& Bers, M. U. (2013). Gender differences in kindergarteners' robotics and programming achievement. International Journal of Technology and Design Education, 23(3), 691-702.

Sullivan, A., Elkin, M., \& Bers, M. U. (2015). KIBO Robot Demo: Engaging young children in programming and engineering. In Proceedings of the 14th International Conference on Interaction Design and Children (IDC '15). ACM, Boston, MA, USA.

Sullivan, A., Kazakoff, E., \& Bers, M. U. (2013). The wheels on the bot go round and round: Robotics curriculum in pre-kindergarten. Journal of Information Technology Education: Innovations in Practice, 12, 203-219. Retrieved from http://www.informingscience.org/Publications/1887 
Wyeth, P. (2008). How young children learn to program with sensor, action, and logic blocks. International Journal of the Learning Sciences, 17(4), 517-550.

\section{Biographies}

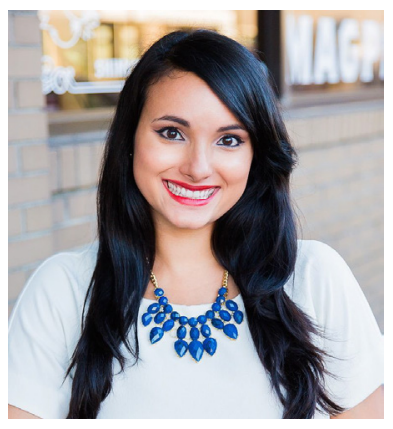

Amanda Sullivan is a researcher at the DevTech Research Group in the Eliot-Pearson Department of Child Study \& Human Development at Tufts University. Amanda is also the Associate Director of the Early Childhood Technology Graduate Certificate Program at Tufts University. She received her Ph.D. and M.A. in Child Development from Tufts University where her dissertation explored young children's development of gender stereotypes about technology and engineering. Amanda's research interests include gender stereotypes, girls and STEM, curriculum development, robotics, programming, STEAM, and the arts.

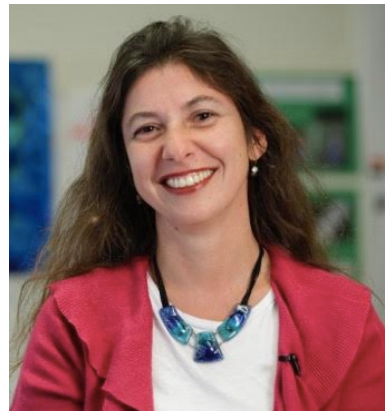

Marina Umaschi Bers is a professor at the Eliot-Pearson Department of Child Study and Human Development and the Computer Science Department at Tufts University. She heads the interdisciplinary DevTech Research group which focuses on designing studying innovative learning technologies to promote positive youth development. Dr. Bers received prestigious awards and has written two books "Blocks to Robots: Learning with Technology in the Early Childhood Classroom" (2008; Teacher's College Press) and "Designing Digital Experiences for Positive Youth Development: From Playpen to Playground" (2012; Oxford University Press). Dr. Bers has an M Ed from Boston University and an MS and PhD from the MIT Media Lab. More on Dr. Bers: http://www.tufts.edu 